\author{
Guillén Ramón, José Manuel. \\ Profesor Universidad Politécnica de Valencia. Centro de Investigación Arte y Entorno.
}

\author{
Del Saz Barragán, Miriam.
}

Investigadora Universidad Politécnica de Valencia, Departamento de Dibujo.

\title{
ACEROGRAFÍA: la plancha acero laminado en frío como soporte alternativo en los procesos de impresión litográficos y en procedimientos mixtos de estampación planográfica, en hueco y relieve.
}

TIPO DE TRABAJO

Comunicación.

PALABRAS CLAVE

Impresión, hierro, litografía, hueco, relieve.

KEY WORDS

Print, iron, litography, engraving.

\section{RESUMEN}

La plancha de acero laminado en frío se ofrece como soporte alternativo en los procedimientos planográficos, en hueco y en relieve, por sus cualidades y por sus posibilidades expresivas. La expresión "Acerografía, es un término nuevo con el que denominamos a este nuevo procedimiento. Etimológicamente procede de los vocablos "acero" y "gráphein".

En el ámbito del grabado, artistas e investigadores continuamente han buscado nuevas posibilidades que enriquezcan el lenguaje gráfico y faciliten la obtención de imágenes. Actualmente podemos constatar los profundos cambios producidos en la obra gráfica, tanto en lo relacionado a factores técnicos como conceptuales y expresivos.

En el año 2000 en colaboración con Daniel Manzano, profesor de la Escuela Nacional de Artes Plásticas de la Universidad Nacional Autónoma de México iniciamos este trabajo de investigación. Actualmente en Valencia, continuamos investigando sobre nuevos recursos y nuevos soportes susceptibles de ser utilizados en los procedimientos litográficos y nos hemos dado cuenta de las ventajas que ofrece la utilización de la plancha de acero laminado en frío en procesos litográficos, así como la posibilidad de interacción con otros sistemas de grabado y estampación en hueco y en relieve. La utilización de este soporte tiene varias ventajas, que describimos a continuación: se encuentran en el comercio fácilmente y son económicas, son fáciles de manipular, su particular estructura facilita el graneado superficial requerido para su utilización en procedimientos litográficos, estas planchas permiten ser estampadas en las prensas litográficas, en el tórculo y en otro tipo de prensas. Es factible integrar procesos de impresión planográficos, en hueco y relieve, utilizando el mismo soporte

Esta investigación ha requerido una labor de documentación importante. A través de un exhaustivo trabajo de campo, analizándose los comportamientos de este material. Se han llevado a cabo diferentes workshops aplicando este proceso en España, Portugal y Polonia.

\section{ABSTRACT}

The cold rolled steel plate is offered as alternative support in lithographic procedures, intaglio and relief, for its qualities and its expressive possibilities. The term "Steelgraphy, is a new term that we call this new procedure. Etymologically derived from the words "steel" and "graphein". 
In the field of printmaking, artists and researchers have continually sought new opportunities to enrich the graphic language and facilitate imaging. Currently we can see the profound changes in the graphic work, both in terms of technical and conceptual and expressive factors.

In 2000, in collaboration with Daniel Manzano, professor of the National School of Plastic Arts of the Universidad Nacional Autónoma de México began this research. Currently in Valencia, continue researching new resources and new media capable of being used in lithographic processes and we have realized the advantages of the use of steel sheet cold rolled in lithographic processes, as well as the possibility of interaction with other systems engraving and intaglio printing and embossing. The use of this support has several advantages, which we describe below: are commercially easily and are inexpensive, they are easy to handle, its particular structure facilitates surface graining required for use in lithographic processes, these plates allow to be stamped lithographic presses, in the printing press and other presses. Is feasible to integrate planográficos printing processes, intaglio and embossed using the same support.

This research has required a important work in documentation. Through an exhaustive fieldwork, analyzing the behavior of this material. They have conducted several workshops applying this process in Spain, Portugal and Poland.

\section{CONTENIDO}

En los sistemas de grabado y estampación en relieve en hueco y planográficos se han producido profundos cambios que han ido acercando progresivamente a los artistas a unos medios con grandes posibilidades de creación y de expresión.

De ser utilizados principalmente como medios de reproducción, adoptados más por artesanos que por artistas, pasan a ser entendidos como medios de creación. Esto ha sido posible no sólo debido a los cambios conceptuales sino a los avances técnicos en el uso de nuevos materiales, soportes, herramientas y procesos. La evolución ha sido constante desde el uso de planchas de madera en los primeros tiempos de la xilografía a los actuales sistemas de grabado y estampación

Consideramos la litografía como un magnífico medio de creación, y la entendemos como un proceso creativo que conlleva una investigación constante, condicionada por criterios técnicos bien definidos y una metodología rigurosa. Como el grabado, supone una actividad racional, ha de pensarse por adelantado, utiliza procedimientos regidos por leyes físicas y químicas que exigen una correcta planificación. Se trata de un medio regido por leyes propias, en muchos aspectos puede considerarse a la vez como fundamento y perfecta expresión de una cultura basada en las ciencias naturales. Sin embargo, dejamos claro que concebimos tanto el grabado como la litografía, no como medios de reproducción, sino como versátiles estrategias de creación, que pueden ofrecer una gran libertad de expresión.

En los últimos años nuestro interés gira en torno a la obra gráfica entendida como hecho creativo, y su carácter de proceso. Actualmente nos encontramos implicados en el estudio de Soportes alternativos a la piedra litográfica y la plancha de aluminio en litografía, centrándonos en la comunicación que presentamos en este congreso, que lleva como título: ACEROGRAFíA: la plancha acero laminado en frío como soporte alternativo en los procesos de impresión litográficos y en procedimientos mixtos de estampación planográfica, en hueco y relieve.

Denominamos "ACEROGRAFÍA" a un nuevo procedimiento basado en el uso de la plancha de acero laminado en frío. Hemos utilizado el término "Acerografía" y no el de "Ferrografía", con el que comenzamos a denominar nuestro procedimiento, pues este último es ya utilizado en la industria para definir una técnica de estudio y análisis de partículas dispersas en aceite para conocer el rendimiento de los lubricantes y máquinas.

Continuando la investigación que ya iniciamos con la colaboración de Daniel Manzano, profesor de la Escuela Nacional de Artes Plásticas de la Universidad Nacional Autónoma de México (UNAM), sobre "Posibilidades de la plancha de hierro laminada en frío y su utilización en procesos de impresión planográfico, en hueco y relieve", queremos poner en evidencia las ventajas que ofrece la utilización de la plancha de acero laminado en frío en litografía, y la posibilidad de interacción con otros sistemas de grabado y estampación en hueco y en relieve. 


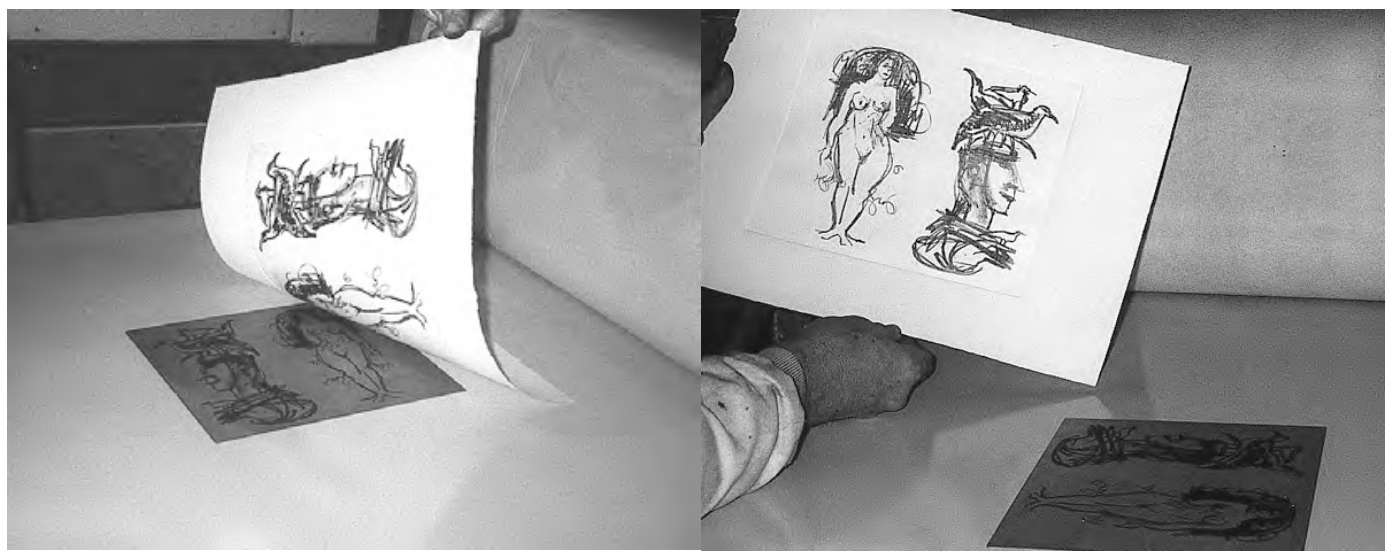

Primeras experiencias realizadas utilizando la técnica litográfica sobre plancha de acero laminada en frío. Obtención de pruebas utilizando el tórculo. Daniel Manzano / José Manuel Guillén. Valencia, 2000

Este proyecto centra principalmente la investigación en un material susceptible de ser usado en litografía, sustituyendo a las matrices tradicionales utilizadas en este medio (piedras litográficas, planchas de zinc y planchas de aluminio). Nuestro objetivo lo llevamos a cabo a través de estudiar a fondo, y analizar la estructura y propiedades de la plancha de acero laminada en frio, así como su reacción y comportamiento en las diferentes técnicas litográficas, para poder ser utilizado como nuevo soporte en este sistema de impresión, además establecer métodos estables en cuanto a la utilización de esta plancha en tres diferentes procesos de impresión, con la convicción de que permite mejoras respecto a los soportes que ya conocemos.

Consideramos que el uso de las planchas de acero laminado en frio supone varias ventajas con relación a las usadas tradicionalmente. Entre otras:

- Son asequibles, se encuentran fácilmente en el mercado laminadas y pulidas. Se comercializan en diferentes formatos y en diferentes grosores. Para nuestra investigación hemos elegido las de 200 × $100 \mathrm{~cm}$ y con un grosor de 0,8 mm La misma distribuidora puede cortarlas con cizalla al formato deseado.

- Su particular estructura, debido al contenido en carbono regularmente repartido, le confiere unas ventajas y unas posibilidades expresivas, que la hacen especialmente aconsejable, facilitando el graneado superficial requerido para su utilización en procedimientos litográficos.

La incomodidad que supone la utilización las pesadas piedras litográficas llevó al propio inventor de la litografía Senefelder, buscar soportes alternativos como el cartón preparado, o posteriormente la plancha de zinc. Actualmente es usada la plancha de aluminio, que requiere ser graneadas por procedimientos laboriosos y no es muy asequible.

- Pueden ser utilizadas tanto en procesos de impresión planográficos, cómo en procesos en hueco y en relieve. Con baños en una disolución de ácido nítrico podemos granearlas nosotros mismos, consiguiendo la textura superficial requerida.

- Posibilitan combinar en una misma estampa y con una sola matriz los diferentes lenguajes que ofrecen los medios planográficos, en hueco y en relieve.

- Permiten ser estampadas con las prensas litográficas, con el tórculo o con otro tipo de prensas.

- Pueden ser recuperadas fácilmente y reutilizadas, cuando son utilizadas en procesos planográficos, lo que indudablemente supone una ventaja económica.

- Permiten fácilmente la realización de trabajos a color a partir de una sola plancha, pudiendo ser recuperada ésta después de la estampación de cada color. Con los procedimientos tradicionales es necesario habitualmente utilizar una plancha para cada color. obra gráfica.

Facilitan la utilización de procedimientos mixtos de grabado y estampación, permitiendo enriquecer las posibilidades de la

- La aplicación de la plancha de acero laminado en frío en procesos de grabado y litografía permite un ahorro considerable a nivel económico en el material empleado en taller. Por esta razón resulta muy útil su aplicación en la enseñanza. 
Esta investigación ha requerido una exhaustiva labor de documentación seguida de un trabajo de experimentación riguroso a través de pruebas y fichas que permitieran analizar los comportamientos de este material, estudiando sus reacciones a la oxidación, los mordientes o la acidulación, propios de la litografía. Queremos conocer las diferentes variables en el uso de estas planchas con el fin de estabilizar sistemas de trabajo desde una perspectiva interdisciplinar.

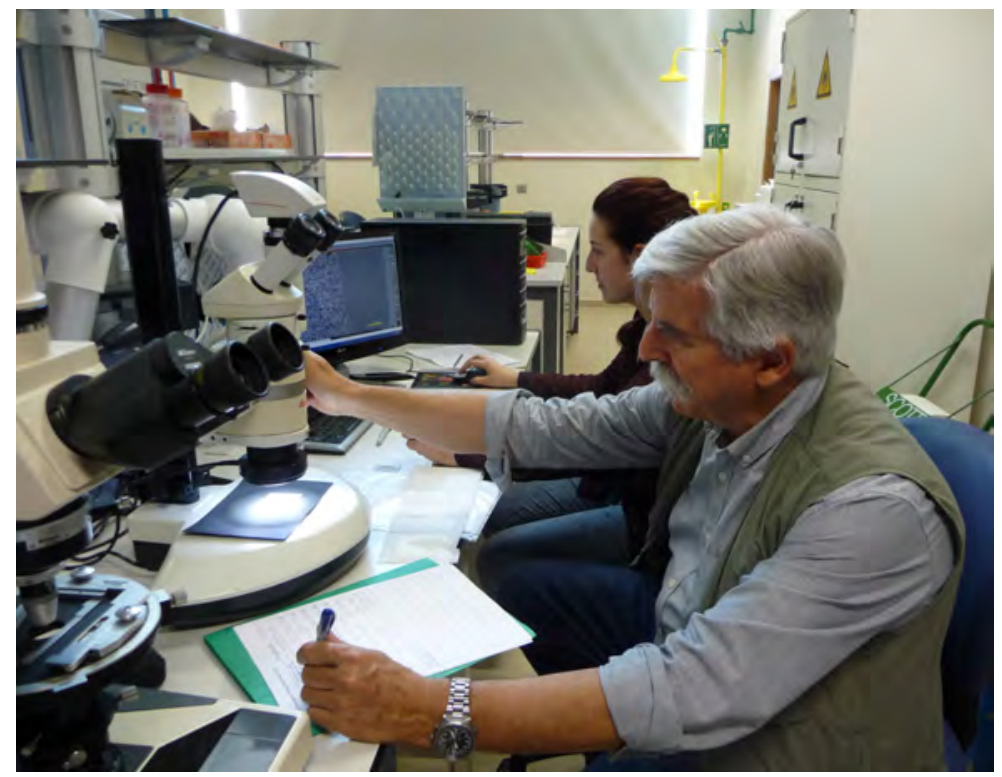

Realización de fichas, analizando el comportamiento de las planchas de acero laminado en frio y su reacción a los diferentes agentes. Servicio de Microscopía Electrónica de la Universidad Politécnica de Valencia. 2015.

La estrategia metodológica planteada ha pretendido en todo momento ser precisa, clara y bien estructurada, adecuada tos objetivos propuestos. Se ha recurrido en una primera fase a los servicios de varios entes investigadores de la Universidad Politécnica de Valencia: el Centro de Investigación Arte y Entorno, CIAE, desde donde se realiza el proyecto, los servicios del Departamento de Ingeniería Mecánica y de Materiales y el Instituto de Tecnología de los Materiales, así como del Servicio de Microscopía Electrónica, instalación dependiente del Vicerrectorado de Investigación de esta universidad. En una segunda fase de investigación y experimentación, comprobando el comportamiento de la plancha de acero en los procesos interdisciplinares de grabado y estampación, se ha recurrido a los talleres y laboratorios de Litografía, Calcografía y Xilografía del Departamento de Dibujo de la Facultad de Bellas Artes de la Universidad Politécnica de Valencia. Complementariamente, hemos desarrollado un trabajo de campo que ha permitido poner en práctica nuestros avances a través de diferentes workshops en España, Portugal y Polonia.
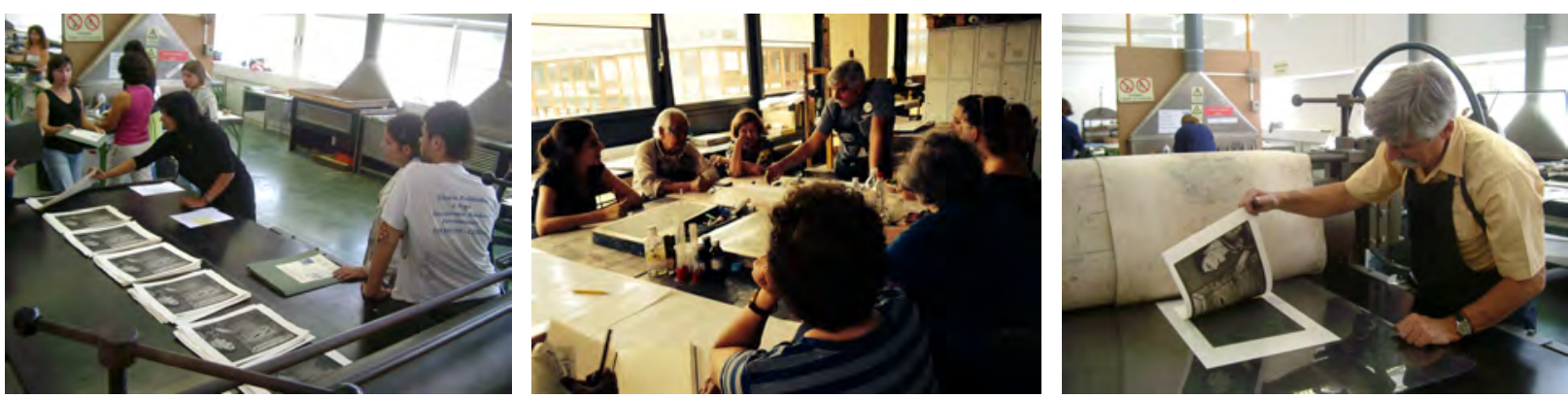

Experiencia y trabajo de campo realizados en la Escola Superior de Artes e Design de Caldas da Rainha. Instituto Politécnico de Leiria (Portugal), 2004 y en The Strzeminski Akademy of Fine Arts. Łódź (Polonia), 2014.

Deducciones sobre las propiedades específicas del acero laminado en frío y su aplicación a los procesos creativos de grabado y estampación planográficos, en hueco y en relieve.

El hierro es un metal dúctil, maleable y muy tenaz, de color gris azulado, magnetizable, que se oxida al aire húmedo; es atacado por casi todos los agentes corrosivos químicos. Prácticamente no se usa en estado puro. En nuestra investigación hemos usado plancha de acero laminado en frio en la que el hierro se encuentra combinado con otros elementos entre los que se encuentra el carbono que mejora su comportamiento. La dureza del hierro es mayor cuanto más proporción de carbono contiene. 
En el mercado podemos encontrar la plancha de acero laminado en frío (también denominada lámina negra), y plancha de hierro galvanizado, esta última con un recubrimiento galvanoplástico de zinc resistente a la oxidación. Este último tipo de plancha no es muy apropiada para ser utilizada, ya que la capa superficial de zinc al ser atacada por el ácido más rápidamente, produce un molesto halo junto en el resultado.

Los principales mordientes que pueden ser utilizados sobre la plancha de acero son el ácido nítrico, el cloruro férrico (percloruro de hierro) y el mordiente holandés. En nuestras experiencias hemos utilizado el ácido nítrico (40 B.), diluido en diferentes concentraciones con agua, su capacidad oxidante lo hace adecuado como mordiente para nuestros propósitos.

Una de las desventajas que posee la plancha de acero laminado frente al zinc o el cobre, es que se oxida con mayor facilidad. Expuesto al aire húmedo se oxida mucho antes que ésos metales. Por ello, es aconsejable conservarla barnizada, engomada o aceitada para preservarla de la oxidación.

\section{La plancha de acero en procesos planográficos}

Conociendo al reacción de la plancha de acero laminado en frío al ser atacada por el ácido, hemos pensado aprovechar esta particularidad, pudiendo aplicar fácilmente una mordida uniforme a la superficie de la plancha previamente desengrasada, para obtener un uniforme graneado superficial que facilitará su uso como soporte alternativo en los procedimientos litográficos.

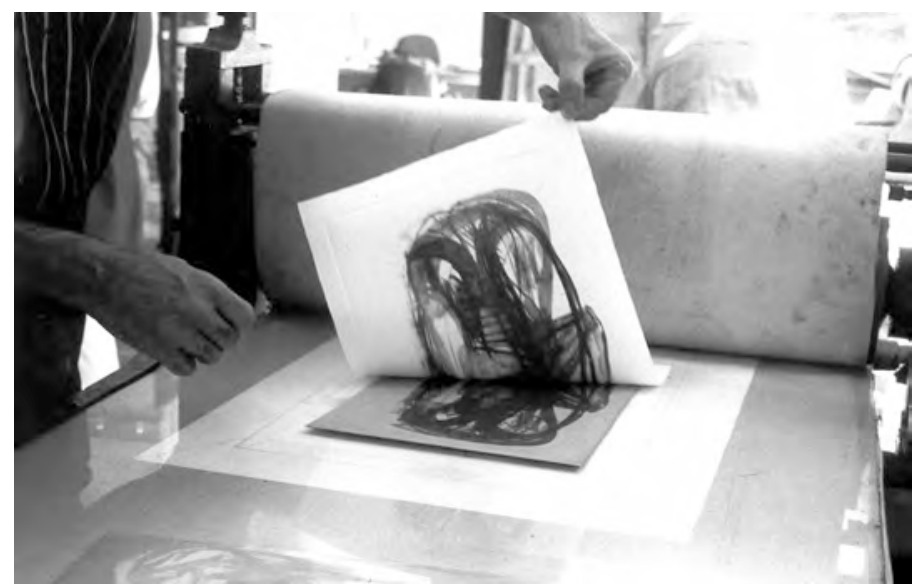

Obtención de una prueba litográfica de un trabajo de Alfonso Sánchez Luna en el workshop realizado en el Instituto Alicantino de Cultura "Juan Gil-Albert" (Alicante), 2003

En litografía, tanto sobre piedra como sobre planchas de zinc o aluminio, es necesario comenzar graneando la superficie. En la piedra el graneado se realiza con borriquete o con otra piedra y abrasivo en una etapa de preparación que es algo lenta y costosa, pero que es más sencilla que la utilización de la graneadora de bolas o los sistemas químicos o electrolíticos necesarios para granear las planchas de zinc o aluminio.

Hemos de decir que hemos pretendido en primer lugar demostrar las ventajas que ofrece la plancha de acero laminado en frío en los procedimientos litográficos, pero buscamos además la interacción entre los diferentes sistemas de grabado y estampación, no limitándonos únicamente a los procedimientos litográficos, ya que podemos recuperar la plancha fácilmente con lijadora eléctrica. para continuar grabándola en procesos en hueco o relieve.

\section{La plancha de acero en procesos en hueco}

Los procedimientos en hueco, o calcográficos, se benefician de la ventaja que ofrece la plancha de acero laminado en frío. Al ser atacada por el ácido, su particular estructura produce un graneado que retendrá fácilmente la tinta de impresión, no necesitando resinar esas zonas evitando las calvas que normalmente se producen en los trazos gruesos cuando éstos son realizados sobre plancha de zinc o de cobre. Con las planchas de acero los trazos ya no tienen limitación de grosor. 


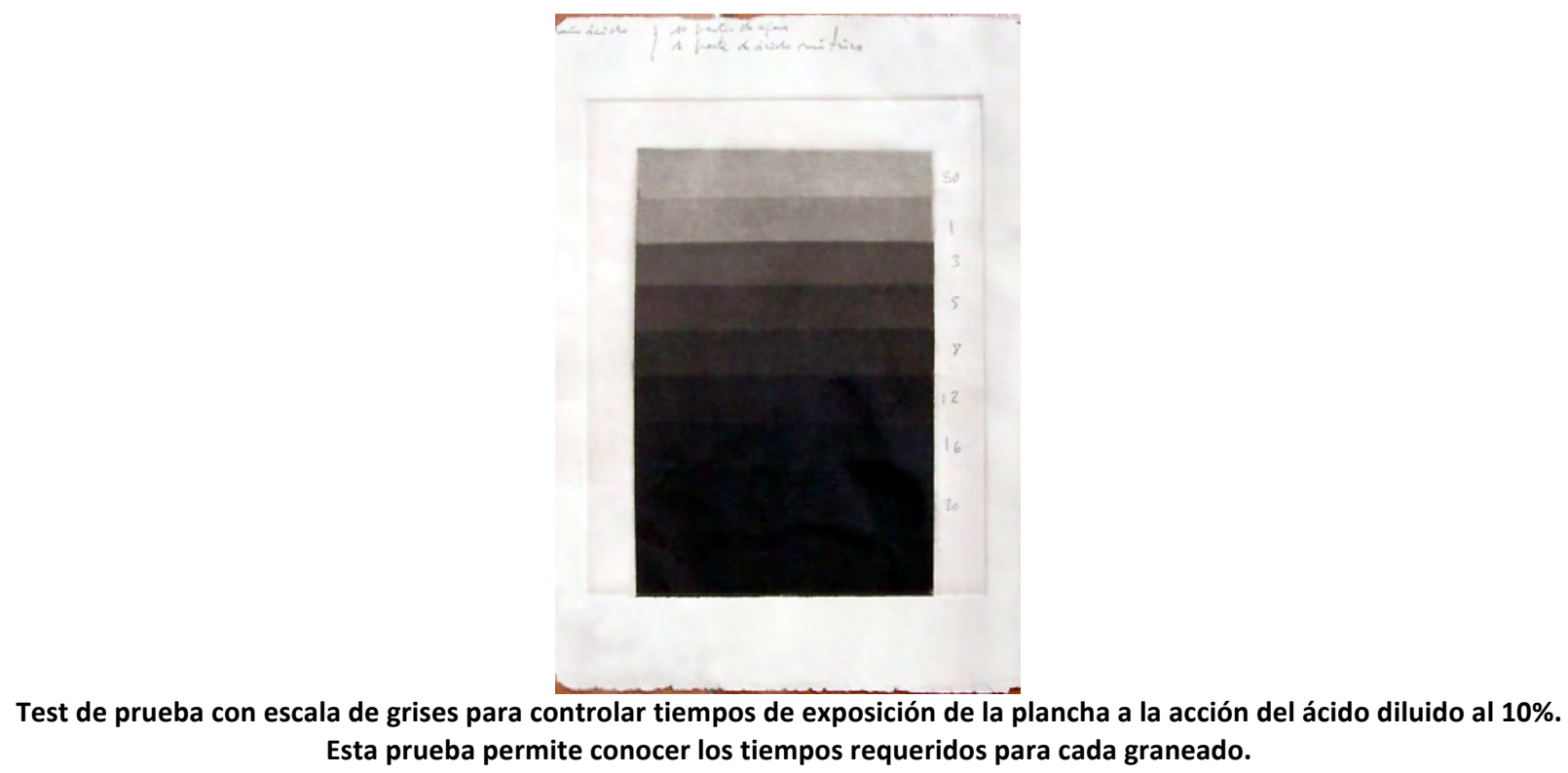

Las técnicas del azúcar, el barniz blando y el aguatinta son perfectamente utilizables sobre plancha de acero. Las aguatintas se pueden realizar directamente sobre el metal sin necesidad de recurrir a aplicar el resinado, una operación delicada y laboriosa que es imprescindible sobre planchas de zinc o cobre. Se pueden obtener graneados con una textura fina y uniforme simplemente utilizando reservas de barniz protector para obtener diferentes tonalidades. Como en las aguatintas en zinc o cobre, es conveniente realizar un test con una escala de grises para controlar las diferentes mordidas de ácido.

Podemos utilizar la técnica del lavis directamente sobre la superficie del metal desengrasado,

aplicando una tinta ácida preparada a base de goma arábiga, ácido nítrico y negro de humo, que aplicaremos con pincel, dejándola actuar más o menos tiempo, de acuerdo a la tonalidad deseada. Siempre es conveniente iniciarnos en esta técnica realizando una prueba de lavis para controlar la capacidad de corrosión de la tinta ácida.

Para la técnica del mezzotinto, no tendremos que recurrir al berceau o al resinado para granear la plancha. Las técnicas directas como el buril o la punta seca en cambio si tendrán más dificultades de realización debido a la dureza del metal.

Dada su microestructura superficial, con la técnica del aguafuerte, las planchas de acero producen un ligero velo de fondo al ser entintadas. Podemos evitarlo puliendo la superficie con una lijadora eléctrica. En la estampación a color con estas planchas podemos obtener colores más puros y mas vivos al no reaccionar con las tintas de color como sucede en las planchas de zinc.

\section{La plancha de acero en procesos en relieve}

La plancha de acero puede ser grabada en profundidad para los procedimientos que requieren un entintado en relieve, con rodillo. Una misma plancha de acero, correctamente grabada, puede ser entintada utilizando al mismo tiempo dos sistemas de entintado: introduciendo tinta en los huecos como en calcografía y entintando con rodillo las zonas superficiales, como en xilografía. Así mismo podemos utilizar la técnica Roll-up de Hayter ${ }^{1}$ para la estampación en color.

\section{La plancha de acero en procesos mixtos}

El uso de estas planchas posibilita utilizar técnicas mixtas y la interacción entre diferentes sistemas de grabado y estampación, no limitándose únicamente a los procedimientos litográficos. La plancha de acero, puede ser elaborada en primer lugar la imagen por procedimiento litográfico, realizar la tirada de esta imagen y recuperar la plancha, esto se realiza fácilmente con una lijadora eléctrica. Podemos posteriormente, mediante unos fáciles registros, continuar elaborando la plancha por el procedimiento del aguafuerte, combinándose de esta forma la expresividad conseguida con los recursos directos los procedimientos litográficos con los matices y texturas que permite el procedimiento calcográfico. Podemos además continuar regrabando la misma plancha en profundidad para integrar en la imagen las calidades que puede ofrecer el entintado superficial con rodillo. Integramos de esta forma, en una misma imagen, y con una sola plancha, las posibilidades que pueden ofrecer cada uno de estos tres sistemas de grabado y estampación: planográfico, hueco y relieve.

\footnotetext{
1 Stanley William Hayter (1901-1988) escribió varios libros y numerosos artículos sobre arte y técnicas gráficas. Desarrolló en el Atelier 17 el método de impresión a color denominado "Roll-up", basado en un sistema de grabado a diferentes profundidades y entintado con tintas de viscosidad variable.
} 


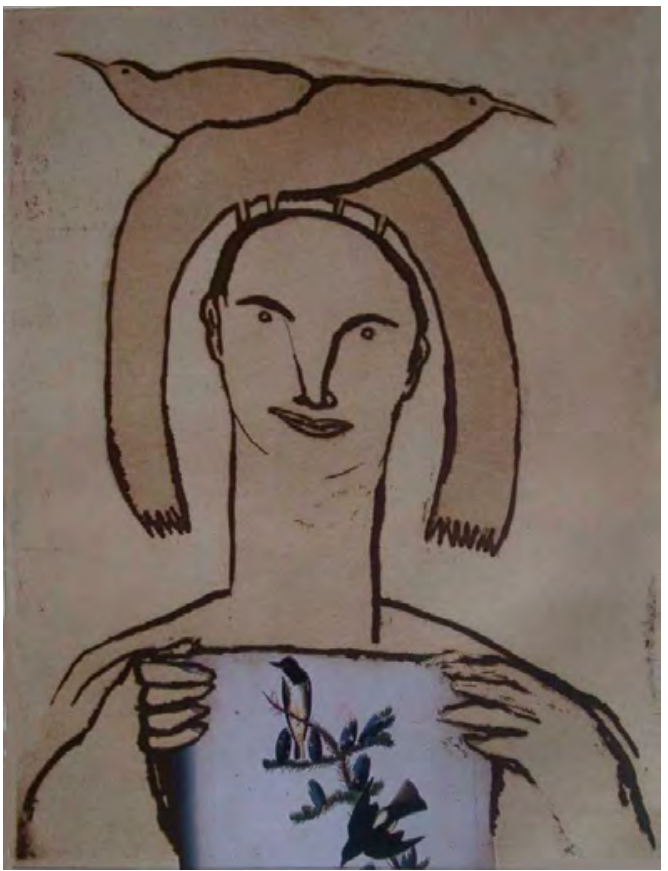

Guillén Ramón. Coleccionista con dos pájaros sobre la cabeza. 2000, (35 x $25 \mathrm{~cm}$ ) Obra realizada sobre plancha de acero laminado en frío, por procedimiento al aguafuerte de trazo y mancha con técnica de goma arábiga tintada y papier collé.

\section{CONCLUSIONES}

El presente trabajo de investigación y las experiencias realizadas, nos llevan a las conclusiones siguientes: nos encontramos con un soporte asequible y alternativo a los habituales en litografía y grabado, permitiendo comenzar un trabajo con procedimientos litográficos, aprovechando la versatilidad del medio y continuar elaborando la misma plancha con procedimientos en hueco, e incluso continuar con procedimientos en relieve integrando en una misma obra las peculiaridades de cada uno de ellos, Consideramos que esta forma de trabajar permite enriquecer nuestro lenguaje gráfico y expresivo.

Este proyecto no queda aquí cerrado, está abierto a futuras investigaciones. Nuestro propósito inmediato es continuarlo a través de la realización de una tesis doctoral que sirva para establecer unos métodos estables, estudiando a fondo la estructura y comportamiento de este soporte, contando con la colaboración de especialistas, desarrollaremos nuevas experiencias que permitan abrir nuevos caminos. Pretendemos que la Acerografía suponga una nueva alternativa que enriquezca las posibilidades de la obra gráfica. 


\section{FUENTES REFERENCIALES}

BARGILLIAT, A. / CAMPBEL, J. Elements des ciences pour les industries graphiques. Institut National des Arts et d'Industries Graphiques. Ministère de l'Education Nationale. París, 1956.

BARGILLIAT, A. Principes Chimiques de la litho et de l'offset. INIAG. París, 1951

BERNARD, J. Métallurgie Générale. Mason et Cie. Editerurs. París, 1969

CASTLEMAN, R. Technics and creativity: Gemini G. E. L. Museum of Modern Art. Nueva York, 1971

DEVON, M. / LAGATTUTA B. / HAMON, R. Tamarind Techniques for Fine Art Lithography, Abrams, 2008

HARTSUCH, PAUL J. Chemistry of Lithography. Lithographic Technical Foundation. Nueva York:, 1952.

HAYTER, S. W. New Ways of Gravure: Innovative Techniques of Printmaking Taken from the Studio of Master Craftsman. Pantheon Books. Nueva York, 1949. Reedit. Oxford University Press. Londres, 1966 y por Watson-Guphill. Nueva York, 1981

HOLMAN, LOUUS.- The Graphic Processes: Intaglio, Relief and Planographic. Goodspeed. Boston, 1929

KUZMIN, B.A. / SAMOJOTSKY, A. I. Metalurgia, metalografía y materiales de construcción. Mir. Moscú, 1986

LAS HERAS Y ESTEBAN, J. M. Tecnología del acero. Ediciones Cedel Barcelona, 1978

MARTIN, E. / TAPIZ, L. Diccionario Enciclopédico de las Artes e Industrias Gráficas. DEAIG. Don Bosco. Barcelona, 1981

MORRAL, F.R. / JIMENO, E / MOLERA, P. Metalurgia general. Tomo I. Reverté S.A. Barcelona, 1982

NEUMAN, THELMA R. Innovative Printmaking. The Making of two-and three-Dimensional Prints and Multiples. Crown Publishers. New York, 1977.

ROSS, J. / ROMANO, C. The Complete Printmaker: the Art Technique of the Relief Print, the Intaglio Print, the Collagraph, the Lithograph, the Screen Print, the Dimensional Print, Photographic Prints, Children's Print, Collecting Prints, Print Worshop. Free Press, Nueva York / Collier Mac Millan. Londres, 1972. Reedit. por John Ross, Clare Romano y Tim Ross, The Free Press. Nueva York, 1991

STOUGHTON, B. The Metallurgie of Iron and Steel. Mc Graw Hill. Nueva York, 1934

VICARY, R. The Thames \& Hudson Manual of Advanced lithography. Thames \& Hudson. Londres, 1977 